\title{
ESCACENA Y DAZA, PIONERO DEL ORIENTALISMO ROMANTICO ESPAÑOL
}

\author{
POR \\ ENRIQUE ARIAS ANGLES \\ Departamento de Historia del Arte del CSIC
}

\begin{abstract}
This article analyzes the early contacts with the orientalism of the sevillan romantic painter José María Escacena y Daza in his relation with the also romantic scotish painter David Roberts.
\end{abstract}

Siguiendo la línea emprendida en dos artículos anteriores, que publicamos en esta revista, sobre los inicios del orientalismo en nuestra pintura romántica, vamos a incidir una vez más en ello, con motivo de haber encontrado datos sobre un pintor sevillano que aportan nueva luz al respecto y nos aclaran más el incierto panorama, hasta el presente, de los comienzos de nuestro orientalismo pictórico.

En el primero de los citados artículos ${ }^{1}$ hacíamos arrancar nuestro orientalismo de Pérez Villaamil, bajo el influjo del pintor escocés David Roberts, a quien aquél conoció durante la estancia de éste en España en 1832-33; mientras que en el segundo ${ }^{2}$ analizábamos, fundamentalmente, la proyección que ese orientalismo de origen británico tuvo, por mediación de Pérez Villaamil, sobre Lucas y deslindábamos la producción orientalista de este último pintor de la de Lameyer. Con ello, concluíamos dando prioridad en su arranque al orientalismo de imaginación sobre el de base en la realidad, ya que ni Pérez Villaamil ni Lucas nos consta que viajasen al mundo oriental.

Pero, mientras que Pérez Villaamil y Lucas no viajaron que sepamos al Oriente, como decimos, estando su orientalismo basado en la pura fantasía e inspirado, total o parcialmente, en los grabados contemporáneos sobre dicha temática -la mayoría de ellos extranjeros- o en algún que otro cuadro de asunto oriental, sin embargo, posteriormente descubrimos que hubo un pintor sevillano que tempranamente viajó a Marruecos, realizando obras orientalistas, inspiradas en asuntos reales, por las mismas fechas en que comenzó a producir Pérez Villaamil

\footnotetext{
${ }^{1}$ Arias Anglés, E.: "Pérez Villaamil y los inicios del orientalismo en la pintura española", Archivo Español de Arte, núm. 281, 1998, p. 1.

${ }^{2}$ Arias Anglés, E.: "Precisiones en torno al orientalismo de Lucas y Lameyer", Archivo Español de Arte, núm. 283, 1998, p. 241.
} 
las suyas. Es decir, que paralelamente al orientalismo de imaginación, y contrariamente a lo que pensábamos, comienza también el que se basa en la más estricta realidad.

Este artista fue el sevillano José María Escacena y Daza (1800-1858), a quien, en justicia, se debe considerar también como uno de los iniciadores del orientalismo pictórico español. Aunque, sin embargo, la poca trascendencia que su pintura tuvo en el panorama general del romanticismo español, reducida al ámbito sevillano, frente a la gran proyección que tuvo, por el contrario, la obra de Pérez Villaamil, hace que su temprano orientalismo de inspiración en la realidad haya quedado relegado frente al imaginativo y fantasioso del gallego, lo que inevitablemente convierte a este último pintor en el candidato más idóneo para ostentar tal título de introductor o promotor de este tipo de representaciones en la pintura española decimonónica.

Poco sabemos de Escacena y Daza, tanto por lo que respecta a su vida como a su obra, ya que son muy pocos los datos biográficos que nos han llegado hasta el presente e igualmente muy escasas las obras que hasta el momento se le conocen, pues raramente aparecen. Por Ossorio y Bernard sabíamos que su fecha de fallecimiento fue la de $1858^{3}$, pero ignorábamos la de su nacimiento. Valdivieso -quizá el único investigador que de él se ha ocupado- nos dice, en una primera publicación en que lo trata, que se desconoce su fecha de nacimiento ${ }^{4}$, pero en otra posterior nos indica ya concretamente que nació este artista en 1800 en Sevilla ${ }^{5}$.

Sabemos también por Ossorio y Bernard que fue alumno de la Escuela de Bellas Artes de su ciudad, en la que consiguió varios premios durante sus estudios, llegando a alcanzar el grado de Teniente Director de la misma en 1829, y el cargo de profesor de la clase de colorido y composición en dicho centro, que ejerció hasta su muerte 6 . Valdivieso nos concreta la fecha en que obtuvo este último puesto de profesor en 1850, año en el que también sitúa su nombramiento como miembro de la Academia de Sevilla ${ }^{7}$, precisándonos y ampliándonos además las noticias aportadas por Ossorio y Bernard, en relación con su aprendizaje en la Escuela de Bellas Artes, al indicarnos que en la citada Escuela hispalense consta que en 1829 y 1830 era alumno de la misma, puesto que en estos años recibió respectivamente el segundo y el primer premio en la clase de Dibujo del Natural ${ }^{8}$.

Igualmente por Ossorio y Bernard sabemos que en la Exposición pública celebrada en Sevilla en 1858, año de su muerte, fue premiado con medalla de plata por un cuadro al óleo que representaba Unos niños jugando y que en la Universal de Londres de 1862 figuraron otros trabajos suyos sobre flores y frutos, asuntos estos que, a decir del citado autor, pintaba con suma gracia . Dado que Escacena murió en 1858, es muy posible que estas últimas obras suyas que se exhibieron en la Exposición Universal de Londres de 1862 fueran allí presentadas por acuerdo de la Academia de Sevilla, pues, sin duda, el reglamento de la citada Exposición Universal contemplaba la posibilidad de que se llevasen obras de artistas recientemente fallecidos, al igual que ocurrió en otros de estos certámenes internacionales.

Según se desprende de estas referencias que hace Ossorio y Bernard a algunas de sus obras y de otras que aporta Valdivieso ${ }^{10}$, parece ser que los géneros pictóricos que cultivó nuestro artista se centrarían en la pintura de costumbres, orientalista, de floreros y bodegones y el retrato.

\footnotetext{
${ }^{3}$ Ossorio y Bernard, M.: Galería biográfica de artistas españoles del siglo XIX, Madrid, 1883-1884, p. 198.

${ }^{4}$ Valdivieso, E.: Pintura sevillana del siglo XIX, Sevilla, 1981, p. 33.

${ }^{5}$ Valdivieso, E.: Historia de la pintura sevillana, Sevilla, 1986, p. 365.

${ }^{6}$ Ossorio y Bernard, M.: Op. cit. nota 3, p. 198.

${ }^{7}$ Valdivieso, E.: Op. cit. nota 4, p. 33; Valdivieso, E.: Op. cit. nota 5, p، 365

${ }^{8}$ Valdivieso, E.: Op. cit. nota 4, p. 33.

${ }^{9}$ Ossorio y Bernard, M.: Op. cit. nota 3, p. 198.

${ }^{10}$ Valdivieso, E.: Op. cit. nota 4, pp. 33, 34; Valdivieso, E.: Op. cit. nota 5, p. 365.
} 
Pero tenemos también noticia de su dedicación- aunque fuese esporádica- a otro género que, por su propia índole, podría relacionarse con lo alegórico, pues el mismo Ossorio y Bernard nos dice además que "Tambien hizo varios trabajos pictóricos imitando á bajo relieves en las exequias celebradas en 1829 en Sevilla por la Reina Doña María Josefa Amalia de Sajonia"”.

Dada la rareza de sus obras, poco podemos precisar con respecto a su pintura en cada uno de los géneros citados que, al parecer, cultivó, pues de alguno no tenemos ni tan siquiera un ejemplo que aportar, tal sería el caso de los floreros y bodegones, teniendo aquí que limitarnos a reseñar la parca opinión que de ellos nos proporciona Ossorio y Bernard, quien nos dice, según ya vimos, que estos asuntos de flores y frutos los pintaba "con suma gracia"12

En cuanto a su dedicación al retrato, Valdivieso nos dice que fue un "sobrio retratista"13, aunque añadiéndonos que los ejemplos que se le conocen en esta especialidad son de secundario interés, citándonos al respecto los retratos de Alonso Cano (Academia de Bellas Artes de Sevilla) y el del canónigo Félix José Reinoso (Biblioteca Colombina, Sevilla), obra ésta realizada en el año de 1852 y que, a juicio del citado autor, presenta escaso interés por ser copia de un original de Gutierrez de la Vega ${ }^{14}$.

Algo más podemos precisar por lo que respecta a su pintura de costumbres -a la que Valdivieso califica como su especialidad artística ${ }^{15}$-, que se halla influida, como la sevillana decimonónica en general, por el costumbrismo de Murillo, tanto en el colorido y composición como en los temas, según parece deducirse de las dos únicas obras de este género que le conocemos y que ambas representan a Pilluelos jugando a los naipes (colección princesa $\mathrm{D}^{\mathrm{a}}$ Esperanza de Borbón, Villamanrique de la Condesa, Sevilla) (Figs. 1 y 2), obras que proceden de la colección de los duques de Montpensier ${ }^{16}$. En estos dos cuadros, aparte los atuendos típicos sevillanos decimonónicos que portan algunos de los pilluelos (como las chaquetillas cortas bordadas y los sombreros calañeses), las mismas figuras de cabezas rapadas y piés descalzos hacia el primer término, sus actitudes (encarando alguno su vista con desparpajo en el espectador), naturalezas muertas (mondas de naranjas por los suelos en el de cuatro personajes y cesta con rábanos en el de dos), así como su naturalismo y dorado e impreciso ambiente, todo ello, como decimos, son elementos aprendidos y tomados del costumbrismo murillesco, al que nos remiten con toda claridad.

Pero, en referencia al asunto que particularmente nos interesa, junto a estos géneros, sabemos, según antes dijimos, que cultivó también la pintura orientalista, como puso de manifiesto Valdivieso, quien cita tres obras suyas de esta temática que aparecen en el catálogo de la colección de los duques de Montpensier, siendo sus títulos Paisaje africano con una tienda de campaña, Pastor árabe y Retrato del Cid Mustaphá el Hasany ${ }^{17}$, obras que, en una primera opinión de dicho investigador, evidencian una estancia de Escacena en Marruecos ${ }^{18}$, mientras que en otra segunda matiza que hacen sospecharla ${ }^{19}$.

\footnotetext{
${ }^{11}$ Ossorio y Bernard, M.: Op. cit. nota 3, p. 198.

${ }^{12}$ Ibidem.

${ }^{13}$ Valdivieso, E.: Op. cit. nota 4, p. 34; Valdivieso, E.: Op. cit. nota 5, p. 365.

${ }^{14}$ Ibidem.

${ }^{15}$ Valdivieso, E.: Op. cit. nota 4, p. 33; Valdivieso E.: Op. cit. nota 5, p. 365.

${ }^{16}$ Valdivieso, E.: Op. cit. nota 4, p. 33; Valdivieso, E.: Op. cit. nota 5, p. 364 (fig. 309) y p. 365 (fig. 310); VV.AA.: Cien años de pintura en España y Portugal (1830-1930), Madrid, 1988, tomo 2, p. 320; Catálogo de los cuadros y esculturas pertenecientes á la galería de SS.AA.RR. los Serenísimos Señores Infantes de España, Duques de Montpensier, Sevilla, 1866, p. 56, núms. 246 y 280.

${ }^{17}$ Catálogo de los cuadros y esculturas pertenecientes á la gelería de SS.AA.RR. los Serenísimos Señores Infantes de España, Duques de Montpensier, Sevilla, 1866, pp. 56, 57, núms. 278, 279 y 282.

${ }^{18}$ Valdivieso, E.: Op. cit. nota 4, pp. 33, 34.

${ }^{19}$ Valdivieso, E.: Op. cit. nota 5, p. 365.
} 

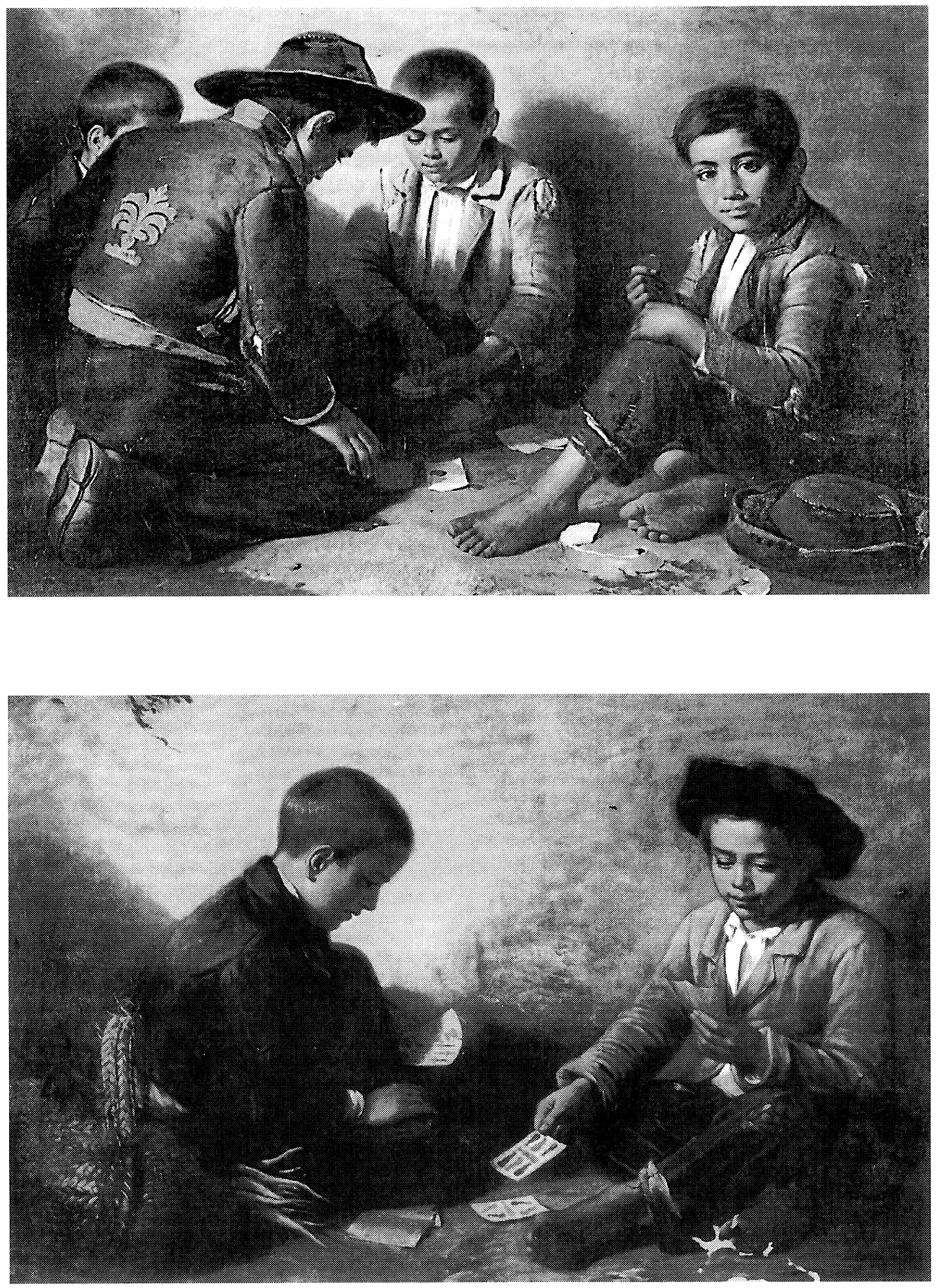

Figs. 1 y 2. JOSE MARIA ESCACENA. Pilluelos jugando a los naipes. Villamanrique de la Condesa (Sevilla). Colección particular. 
Por su parte, Dizy, aumenta el catálogo orientalista del pintor publicando y reproduciendo dos obras suyas, de colección particular, de asunto claramente marroquí, que son las primeras de temática orientalista que podemos ver de este artista.

Una de ellas, que el autor titula Ante una casa, Marruecos ${ }^{20}$ (óleo/panel, 0,46 x 0,36 ms., firmado en el ángulo inferior derecho: "J. ESCAZENA FT. GIBRALTAR") (Fig. 3), representa a un moro sentado ante el portal de una casa, de indudable estilo marroquí, y al que se acerca una mujer, viendose tras ella, en la calle, a otros dos personajes charlando; las vestimentas de todos ellos son claramente atuendos marroquíes.

La otra, a la que Dizy titula $A$ la entrada de un café marroquî́ (óleo/panel, $0,46 \times 0,36 \mathrm{~ms}$., firmado en el ángulo inferior izquierdo: "ESCAZENA FT. GIBRALTAR") (Fig. 4), creemos que no responde a este asunto, sino que más bién parece se trata de la casa de un santón, jefe o juez, personaje semidesnudo que se halla al fondo de una estancia, departiendo sentado en actitud autoritaria con otros moros, uno de los cuales sostiene un papel en sus manos, y a los que se acerca un criado o esclavo negro portando un sevicio de té, encontrándose, al primer término, ante un portalón, un guardián con espingarda; igualmente, tanto los atuendos de los personajes como las arquitecturas, son marroquíes.

En principio, las sospechas de una estancia en Marruecos de Escacena, no quedarían más que en eso, en mera suposición, pues tanto los asuntos que cita Valdivieso, como los que reproduce Dizy, bien pudieran estar tomados de grabados, y aunque los de este último, por su claro carácter marroquí, pudiesen inducir a sostener más firmemente la posibilidad de dicha estancia, la realidad es que el citado autor nada dice al respecto, limitándose a constatar, mediante los títulos que da a los cuadros, que son asuntos marroquíes, pero sin indicar su carácter bien meramente imaginativo o con base en la realidad ${ }^{22}$.

Sin embargo, nosotros hemos podido comprobar lo acertado de las sospechas de Valdivieso. Se trata de un documento, que publicamos en su día, que nos demostraba que existió un conocimiento personal entre Pérez Villaamil y David Roberts en la ciudad de Sevilla ${ }^{23}$; y aunque, en principio, también confirmaba una estancia de Escacena en Marruecos, no le prestamos entonces ninguna atención al respecto, por ser asunto este último totalmente ajeno a nuestras investigaciones del momento, centradas entonces en Pérež Villaamil, pero que ahora nos sirve también para aclarar el problema que tratamos.

En efecto, se trata de un documento de la Real Academia de Bellas Artes de San Fernando, fechado el 20 de enero de 1851, en el que el profesor José María Escacena y Daza comunica al Secretario General de dicha Academia que ha remitido a la misma desde Sevilla, por medio de los académicos de número de la misma Antonio María Esquivel y Jenaro Pérez Villaamil, cuatro pinturas suyas que somete al exámen de esa corporación ${ }^{24}$; no sabemos con motivo de qué, pues no se nos indica, aunque quizá fuese en razón de algún nombramiento al que aspiraba o a su presentación a alguna exposición.

Pero lo interesante de dicha comunicación es que, por un lado, nos confirma también el conocimiento personal y las relaciones profesionales entre Escacena y David Roberts al indicarnos claramente que una de las pinturas que envía "la que representa un retrato de cuerpo entero fue pintada por mi en 1833 y el fondo de ella ejecutado por el célebre pintor inglés David Roberts, segun consta á los dos citados Sres. Academicos"25; y por el otro, nos corro-

\footnotetext{
${ }^{20}$ Dizy Caso, E.: Los orientalistas de la escuela española, París, 1997, p. 80.

${ }^{21}$ Ibidem, pp. 80, 81.

${ }^{22}$ Ibidem, p. 80.

${ }^{23}$ Arias Anglés, E.: El paisajista romántico Jenaro Pérez Villaamil, Madrid, 1986, pp. 47, 48.

${ }^{24}$ Ibidem, p. 508 (documento núm. 193).

${ }^{25}$ Ibidem.
} 

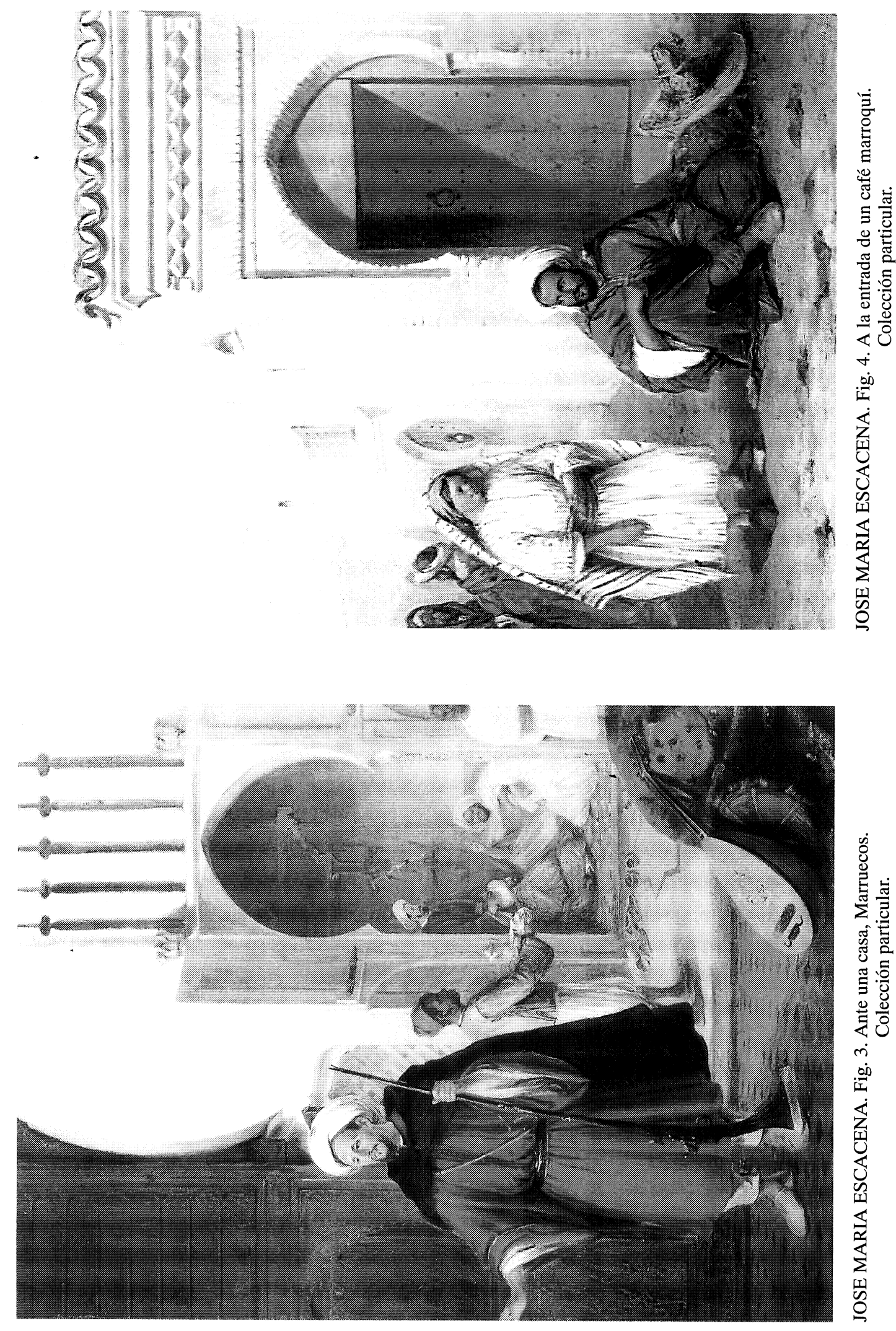
bora también una estancia en Marruecos de Escacena al volvernos a indicar, refiriéndose a otra de las pinturas, que "fue pintada por mi en Tanger en 1834 y representa dos gefes Arabes" 26 .

Con ésto creemos queda suficientemente demostrado un viaje de Escacena a Marruecos y en fecha tan temprana, para la cronología de nuestro orientalismo, como la de 1834, año en el que practicamente comienzan a aparecer las primeras obras orientalistas de reivindicación arqueológica, en consonancia con el pensamiento historicista europeo, de Pérez Villaamil, conocido también de Escacena, como, a la vez, David Roberts lo fue de ambos.

Por otro lado, y poniendo en conexión ambas noticias, podemos preguntarnos si no jugaría algún papel en este viaje a Marruecos de Escacena el posible influjo de David Roberts, dadas las fechas en que lo realiza.

En efecto, Roberts viajó por España desde diciembre de 1832 hasta septiembre de 1833. El 16 de diciembre de ese primer año llegó a Madrid, viniendo por Vitoria y Burgos; el 30 de enero de 1833 estaba en Córdoba; a mediados de febrero en Granada; a principios de marzo en Málaga; a finales de este mes en Tánger; en abril visita Cádiz, Jerez y Gibraltar, y escribe desde Sevilla el 4 de mayo. Aquí, la ciudad, los amigos y las ocasiones le retienen, retrasando su proyectado regreso a través de toda España. Durante los meses que permanece en Sevilla trata a los pintores de la ciudad, escribe sobre ellos y realiza también pinturas. El 4 de septiembre de 1833 aún está en la capital andaluza, fecha en la que escribe a su amigo Hay diciéndole que el cólera se había extendido de Portugal a Sevilla, con lo que abandona la idea de recorrer España y decide marchar inmediatamente por barco ${ }^{27}$.

Pues bien, según ese itinerario, vemos que Roberts estuvo en Tánger a finales de marzo de 1833 y Escacena nos dice que su cuadro representando a dos jefes árabes lo había pintado en Tánger en 1834, pero también nos especifica que el fondo de su otro cuadro lo había pintado Roberts en 1833, lo que indica, según hemos dicho, un conocimiento y estrecha relación entre ambos en este último año, en el que Roberts había pasado a Tánger. Por ello, no podemos evitar la tentación de aventurar la posibilidad de que Escacena acompañara a Roberts en su corta excursión al norte de África y más cuando los dos cuadros đe asuntos marroquíes publicados por Dizy, antes citados, están firmados por Escacena en Gibraltar y, como hemos visto, Roberts estuvo en Gibraltar a finales de abril de ese año de 1833.

Pudiera ser, como apuntamos, que Escacena acompañara a Roberts en su visita a Tánger y que, animado por lo que allí viera, repitiera el viaje, ya él solo, en 1834, año y población en que dice realizó su cuadro representando a dos jefes árabes. Sin embargo, pudiera ser también que no acompañase a Roberts a Tánger en 1833, pero que, animado por el escocés y por los apuntes que éste de allí trajese, se decidiese él a seguir sus pasos, visitando Tánger en 1834, fecha en la que afirma que pintó en esta ciudad el citado cuadro de los dos jefes árabes, suposición ésta que parece la más plausible.

Sea como fuere, el hecho incuestionable es que Escacena estuvo en Marruecos en 1834 y que fruto de este viaje -o además de otro posible anterior acompañando a Roberts- fueron la realización de toda una serie de pinturas con asuntos marroquíes, de las que son testimonio las seis aquí citadas (las tres que menciona Valdivieso, la que nos proporciona el propio Escacena y las dos que publica Dizy), quizá como punta de

\footnotetext{
${ }^{26}$ Ibidem.

${ }^{27}$ Ibidem, pp. 45, 46; Salas, X. de: “Varias notas sobre Jenaro Pérez Villaamil”, Archivo Español de Arte, t. XXXI, 1958, pp. 280, 281, 284; Ballantine, J.: The life of David Roberts R.A. compiled from his journals and other sources by...Edinburgh, 1866; Bruce Thomson, J.: “The late David Roberts R.A.”, Macmillans Magazine, núm. 8, noviembre de 1866 , p. 39 y sigs.
} 
iceberg de su desconocida producción en general y de su temática orientalista en particular.

Así pues, a la par que el orientalismo de fantasía introducido en nuestra pintura por Pérez Villaamil, se va a dar también entre nosotros, en los mismos inicios de la época romántica, y aunque sólo sea reducido al concreto caso de Escacena, el orientalismo que buscó directa inspiración en el natural, acercándose al asunto "in situ", mediante la realización del viaje -en cierto modo iniciático- al Oriente.

Ésto, que sería más frecuente después entre los orientalistas españoles, concretamente a partir de la guerra de África de 1859-1860, es lo que hace de Escacena un precursor. Y si precisamente David Roberts fue uno de los pioneros románticos de dicho viaje, es innegable que le siguió prontamente en ésto un pintor español, que si bien no tuvo ni su proyección ni su trascendencia, no deja por ello de tener importancia entre nosotros, por constituir claro ejemplo del pintor romántico viajero al Oriente, tan en boga en la Europa romántica, con el que así se incorpora también prontamente España a dicha moda y corriente de carácter internacional.

En cuanto al estilo que manifiestan los dos únicos cuadros que le conocemos a Escacena de asuntos orientalistas (los publicados por Dizy que más arriba citamos), hemos de decir que se apartan del influjo murillesco que parece dominó en su pintura costumbrista, según ya vimos. Frente a los tonos dorados, contrastadas luces y sombras, fuerte sentido del volumen y acusado naturalismo de tradición española, que predominan en los dos cuadros de pilluelos sevillanos, estos otros dos cuadros orientalistas de Escacena poseen una mayor luminosidad (sobre todo el cuadro de exterior), y claridad del colorido, desapareciendo los apagados tonos y los fuertes contrastes lumínicos que dominan en aquellos otros, estando también las figuras concebidas con un sentido menos contundente; una imaginería más ligera envuelta en una claridad lumínica y una suavidad cromática que nos recuerdan a las de la pintura a la acuarela y las concepciones orientalistas de los pintores románticos británicos que por aquellas fechas viajaron a España, tanto de Roberts como de Lewis, pero sin llegar, desde luego, a la finura y calidad de ellos.

Por lo que respecta a Roberts, nada de extraño tiene ésto, sabiendo, como sabemos, la relación profesional que debió de existir entre Escacena y el pintor escocés mientras éste estuvo en Sevilla, ya que llegaron a pintar incluso juntos (casi dirîamos que al alimón) uno de los cuadros que Escacena presenta a la Academia de San Fernando en 1851, según el propio testimonio de éste, quien afirma que el fondo de ese retrato suyo fue pintado por David Roberts, como ya vimos. Y ésto implica, sin duda, un conocimiento de la pintura de Roberts por parte de Escacena.

Pero, por otro lado, la claridad de estas dos pinturas de Escacena y la ligereza de las figuras representadas, nos recuerdan también (aunque sea someramente) a la concepción acuarelística de Lewis y a su fina imaginería, sin llegar, como decimos, a alcanzar su calidad, elegancia y sutileza, así como igualmente nos remiten a esa visión más naturalista del asunto oriental, liberada de artificios dramáticos y basada en una observación aguda de la vida cotidiana de ese mundo, que tuvo Lewis frente a la más romántica de Roberts ${ }^{28}$.

Por todo ello, podríamos atrevernos incluso a sospechar un posible conocimiento entre Escacena y el pintor inglés John Frederick Lewis, quien, al igual que Roberts y por las mismas fechas, entre 1832 y 1834, estuvo viajando también por España y Tánger. Si bien durante este viaje Lewis evitó encontrarse con su amigo Roberts, sin que sepamos exactamente por qué, a pesar de las cartas que le escribió éste último invitándole a ello, llegando incluso a cruzarse sus caminos, sin que coincidieran, en su muy similar itinerario ${ }^{29}$, el hecho de que Lewis

\footnotetext{
${ }^{28}$ Peltre, Ch.: Les orientalistes, París, 1997, p. 133.

${ }^{29}$ Ackerman, G.M.: Les orientalistes de l'école britannique, París, 1991, p. 182.
} 
utilizara la residencia sevillana del escritor y dibujante aficionado inglés Richard Ford -que entonces vivía en esta ciudad con su familia- como apeadero suyo en este viaje ${ }^{30}$, podría darnos pié para ello. De hecho, lo sabemos con seguridad residiendo en casa de Ford en Sevilla desde diciembre de 1832 hasta marzo de 1833, aproximadamente ${ }^{31}$.

Como decimos, estas circunstancias, tan similares a las de David Roberts, y el hecho de que Richard Ford fuera personaje muy conocido en Sevilla e introducido en los mejores ambientes, incluido el artístico, todo ello unido al estilo claro y de delicadas figuras de los dos cuadros orientalistas que le conocemos a Escacena, son elementos que nos tientan a aventurar esta suposición que, por otro lado, dado el reducido círculo artístico sevillano del momento, no tendría por qué parecer excesivamente descabellada.

Pero, al márgen de que Escacena y Lewis llegasen o no a conocerse, el hecho incuestionable es el influjo que algunos de estos pintores románticos británicos ejercieron sobre los españoles en los mismos inicios de nuestro romanticismo, particularmente en Andalucía, aspecto éste al que ya dedicamos un estudio en su día ${ }^{32}$, y que podemos constatar, una vez más, y reforzar con el particular caso de Escacena, que se sumaría así a los ejemplos entonces aportados.

Estas influencias no se limitarían solamente a aspectos estilísticos (tal sería el caso de Pérez Villaamil respecto a Roberts), sino que el trato con estos pintores extranjeros actuó incluso como acicate en los españoles, despertando en ellos actitudes románticas europeas y deseos de emulación, lo que queda perfectamente reflejado, en el caso de Escacena, en su temprano interés por el orientalismo y en su viaje a Marruecos tan inmediato al de Roberts, siguiendo en ello, sin duda, las huellas de estos románticos británicos citados, especialmente las de este último.

\footnotetext{
${ }^{30}$ Ibidem.

${ }^{31}$ Ford, B.: Richard Ford en Sevilla, Madrid, 1963, p. 18.

${ }^{32}$ Arias Anglés, E.: "Influencia de los pintores ingleses en España" en Imagen romántica de España, Ministerio de Cultura, Madrid, 1981, p. 79 y sigs.
} 